\title{
Stimulated Raman gain scattering in thin planar dielectric waveguides
}

\author{
Johannes S. Kanger, Cees Otto, and Jan Greve \\ Applied Optics Group, Department of Applied Physics, BMTI and MESA Institutes, University of Twente, \\ P.O. Box 217, 7500 AE Enschede, The Netherlands
}

Received May 10, 1995

\begin{abstract}
The stimulated Raman gain effect in planar dielectric waveguides is analyzed for the study of thin layers. Calculations show high gain factors and predict the possibility of detecting monolayers. Compared with those for methods based on reflection, the gain can be 4 orders of magnitude higher for a monolayer of benzene. It is concluded that waveguide stimulated Raman gain spectroscopy is a promising technique for the study of thin layers. (c) 1995 Optical Society of America
\end{abstract}

The study of conformational properties of very thin layers or monolayers is difficult. The usual spectroscopic techniques do not have enough sensitivity, a reason why alternative techniques have been developed. The combination of spectroscopy and waveguides seems to be especially promising in this respect. The combination of dielectric waveguides and spontaneous Raman spectroscopy yields, for example, a useful and sensitive method with which to analyze thin layers present on top of these waveguides. ${ }^{1}$ Spectra of several polymer waveguides and of thin layers of polymers on top of a glass waveguide have been measured. ${ }^{1}$ It was shown by Stegeman et al. that coherent anti-Stokes Raman spectroscopy (CARS) also can be combined with waveguides ${ }^{2}$ (WCARS). Very high CARS signal levels were predicted and measured for polystyrene waveguides. ${ }^{3}$ Monolayers on top of a waveguide have also been measured ${ }^{4-6}$ by nonresonant background suppression schemes based on destructive interference among guiding modes for pump, Stokes, and CARS beams. $^{2,7}$ In this Letter stimulated Raman gain spectroscopy in waveguides (WSRGS) is analyzed. Large gain factors are predicted. As with Raman spectroscopy and CARS, stimulated Raman gain allows for the measurement of vibrational spectra. Given these spectra, a detailed analysis of band intensities, positions, widths, and depolarization ratios can provide information about the conformational properties of the molecules. ${ }^{8}$ Because stimulated Raman scattering does not suffer from the intense nonresonant background from the waveguide, which cannot always be fully suppressed in WCARS measurements, WSRGS could be a promising alternative technique.

The waveguide considered is a planar dielectric layered structure consisting of three layers, as shown in Fig. 1. The top layer is the cladding, the lower layer is the substrate, and the layer between is the film. Planar waveguides can guide TE modes (polarized parallel to the $y$ direction) as well as TM modes (polarized perpendicular to the $y$ direction). The electric field for a TE mode with frequency $\omega$ can be expressed as

$$
\mathbf{E}^{k}=\frac{1}{2} \mathbf{e}_{y} \exp \left[j\left(\omega t-\beta^{k} x\right)\right] f^{k}(z) a^{k}(x)+\text { c.c. },
$$

where $\beta^{k}$ denotes the propagation vector of the mode, $f^{k}(z)$ the electric-field distribution function, $a^{k}(x)$ the amplitude distribution function, and c.c. the complex conjugate. The modes are enumerated by $k$. Using the matrix method given by Ghatak et al., ${ }^{9}$ we can calculate the electric-field distribution functions $f^{k}(z)$ numerically. For a $\mathrm{TE}_{0}$ and a $\mathrm{TE}_{1}$ mode these functions are depicted in Fig. 1. The functions $f^{k}(z)$ are normalized such that $\left|a^{k}(x)\right|^{2}$ gives the guided power per meter wave front (in the $y$ direction) in watts per meter.

The theory of third-order nonlinear processes in thin-layer waveguides was derived by several authors. ${ }^{10,11}$ The induced polarization describing the stimulated Raman process is given by the third-order polarizability ${ }^{12}$ :

$$
\begin{aligned}
\mathbf{P}^{(3)}= & \overline{\bar{\chi}}^{(3)}\left(\omega_{\text {Stokes }} ; \omega_{\text {pump }}, \omega_{\text {Stokes }},-\omega_{\text {pump }}\right) \\
& : \mathbf{E}_{\text {pump }} \cdot \mathbf{E}_{\text {Stokes }} \cdot \mathbf{E}_{\text {pump }}^{*} .
\end{aligned}
$$

The third-order susceptibility tensor is denoted by $\overline{\bar{\chi}}^{(3)}\left(\omega_{\text {Stokes }} ; \omega_{\text {pump }}, \omega_{\text {Stokes }},-\omega_{\text {pump }}\right)$, which is a fourth-rank tensor with components $\chi_{i j k l}^{(3)}$ with $i, j, k$, and $l$ one of the Cartesian coordinates $x, y$, or $z$. For convenience, only TE modes are considered here. In this case the induced polarization $\mathbf{P}^{(3)}(z, x)$ can be written as

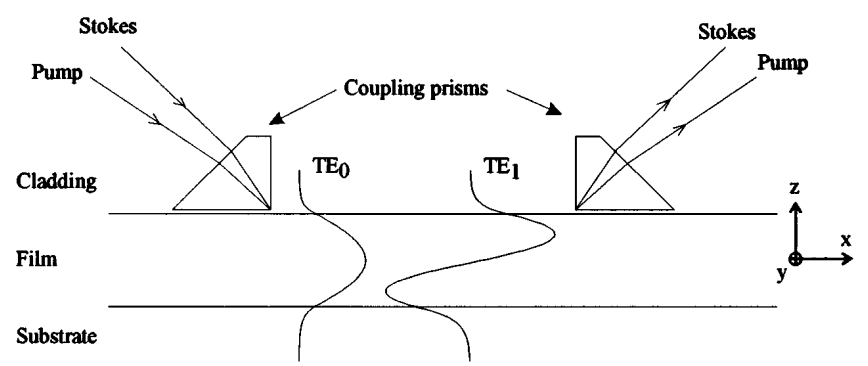

Fig. 1. Waveguide configuration consisting of cladding, film, and substrate. The pump and the Stokes beams are coupled into the waveguide by means of a prism to excite specific modes. The mode distribution functions of a $\mathrm{TE}_{0}$ mode and a $\mathrm{TE}_{1}$ mode are also shown. 


$$
\begin{aligned}
\mathbf{P}^{(3)}(z, x)= & \bar{\chi}^{(3)}(z): \mathbf{E}_{0, \text { pump }}^{k}(z) \mathbf{E}_{0, \text { Stokes }}^{l}(z) \mathbf{E}_{0, \text { pump }}^{k^{*}}(z) \\
& \times\left[a_{\text {pump }}^{k}(x)\right]^{2}\left[a_{\text {Stokes }}^{l}(x)\right] \exp \left(-j \beta_{\text {Stokes }}^{l} x\right),
\end{aligned}
$$

with

$$
\begin{aligned}
\mathbf{E}_{0, \text { pump }}^{k}(z) & =\frac{1}{2} \mathbf{e}_{y} \exp \left[j\left(\omega_{\text {pump }} t\right)\right] f_{\text {pump }}^{k}(z), \\
\mathbf{E}_{0, \text { Stokes }}^{l}(z) & =\frac{1}{2} \mathbf{e}_{y} \exp \left[j\left(\omega_{\text {Stokes }} t\right)\right] f_{\text {Stokes }}^{l}(z) .
\end{aligned}
$$

The induced polarizability will radiate in all possible guiding and radiating modes. Since we are describing stimulated Raman gain spectroscopy, only the Stokes mode $l$ will be considered. Using coupled-mode theory we find for the amplitude dependence on $x$ for the Stokes mode $l$ :

$$
\begin{aligned}
\frac{\mathrm{d}}{\mathrm{d} x}\left(a_{\text {Stokes }}^{l}\right)= & -\frac{j \omega_{\text {Stokes }}}{4} \exp \left(j \beta_{\text {Stokes }}^{l} x\right) \\
& \times \int_{-\infty}^{\infty} \mathbf{P}^{(3)}(z, x) \cdot\left[\mathbf{E}_{0, \text { Stokes }}^{l}(z)\right]^{*} \mathrm{~d} z .
\end{aligned}
$$

Substitution of Eq. (3) into Eq. (5) then leads to

$$
\frac{\mathrm{d}}{\mathrm{d} x}\left(a_{\text {Stokes }}^{l}\right)=\frac{\omega_{\text {Stokes }}}{4}\left[a_{\text {pump }}^{k}(x)\right]^{2}\left[a_{\text {Stokes }}^{l}(x)\right] F^{k l},
$$

where the overlap integral $F^{k l}$ is given by

$$
F^{k l}=-\int_{-\infty}^{\infty} j \chi_{y y y y}^{(3)}(z)\left[f_{\mathrm{pump}}^{k}(z)\right]^{2}\left[f_{\text {Stokes }}^{l}(z)\right]^{2} \mathrm{~d} z
$$

and contains all the information about the waveguide structure and applied modes.

We can now easily calculate the amplitude of the Stokes mode by integrating Eq. (6) from $x=0$ to $x=L$, where $L$ is the interaction length between the Stokes and the pump beams. Solving this integral results in

$$
a_{\text {Stokes }}^{l}(L)=a_{\text {Stokes }}^{l}(0) \exp \left[\left(\omega_{\text {Stokes }} / 4\right)\left(a_{\text {pump }}^{k}\right)^{2} F^{k l} L\right] .
$$

The power of the Stokes mode $l$ can now be calculated. When the beams inside the waveguide have a width $H$, the results can be expressed in terms of the total power in the pump and the Stokes beams:

$$
\begin{aligned}
P_{\text {Stokes }}^{l} & =P_{\text {Stokes }}^{l}(0) \exp \left[\left(\omega_{\text {Stokes }} / 2\right) P_{\text {pump }}^{k} \operatorname{Re}\left(F^{k l}\right) L / H\right] \\
& =P_{\text {Stokes }}^{l}(0) \exp (g L)
\end{aligned}
$$

with $G_{0}$ the total gain given by

$$
G_{0}=g L=\left(\omega_{\text {Stokes }} / 2\right) \operatorname{Re}\left(F^{k l}\right) P_{\text {pump }}^{k} L / H .
$$

From Eq. (10) it can be seen that only the real part of the overlap integral contributes to the total gain of the Stokes beam. This indicates that [see Eq. (7)] only the imaginary part of the third-order susceptibility $\chi^{(3)}$ is responsible for the gain. Since the nonresonant part of $\chi^{(3)}$ is real (far from resonance) it means that the observed signal contains only resonant information and does not suffer from nonresonant background. Using the derived formulas, we will show numerical results obtained for a few experimental situations.

As an example a polystyrene $\left(n_{\text {film }}=1.6\right)$ waveguide on top of a $\mathrm{SiO}_{2}\left(n_{\text {substrate }}=1.46\right)$ substrate is considered. The cladding is air $\left(n_{\text {cladding }}=1\right)$. Polystyrene has $\chi^{(3)} \sim 10^{-12}$ esu (Ref. 13) for the benzene ring stretch vibration (Raman shift of $998 \mathrm{~cm}^{-1}$ ). The width of the beams is $100 \mu \mathrm{m}$, and the power of the pump beam inside the waveguide is fixed at $10 \mathrm{~W}$. For a waveguide with a thickness of $1 \mu \mathrm{m}$ this corresponds to an intensity of $\sim 10 \mathrm{MW} / \mathrm{cm}^{2}$ in the waveguide, which is well below the damage threshold. ${ }^{3}$ These powers are easily available from mode-locked picosecond laser systems. For pulses of 5-ps width and $4-\mathrm{MHz}$ repetition rate the average power is only $\sim 0.2 \mathrm{~mW}$. When the beams are arranged collinearly, long interaction lengths are possible in waveguides. In this particular situation an interaction length of $1 \mathrm{~cm}$ is assumed. A $\mathrm{TE}_{0}$ guiding mode is used for the pump as well as for the Stokes beam $(l=m=0)$. The result is shown in Fig. 2. The gain $G_{0}$ is plotted on the vertical axis as a function of the polystyrene layer thickness. The function depends strongly on the layer thickness and peaks at $\sim 0.35 \mu \mathrm{m}$. This thickness corresponds to the highest density of the optical fields inside the polystyrene layer. When the thickness is smaller the electric field penetrates more into the cladding and the substrate, leading to a smaller density of the optical fields. For a thicker polystyrene layer the electric field becomes located more inside the polystyrene layer but the density of the optical field decreases because the pump power is fixed at $10 \mathrm{~W}$. This all leads to an optimum, as shown in Fig. 2. The calculated maximum gain $G_{0}=0.021$ is high for such thin layers and can be easily detected.

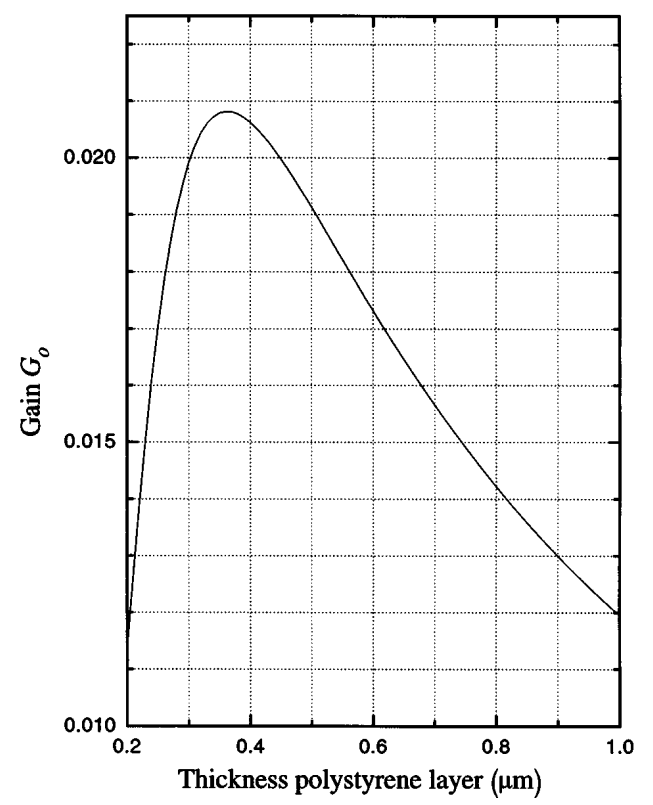

Fig. 2. Gain coefficient $G_{0}$ as a function of the polystyrene layer thickness. The pump power in this case is $10 \mathrm{~W}$, and the interaction length $L=1 \mathrm{~cm}$. 


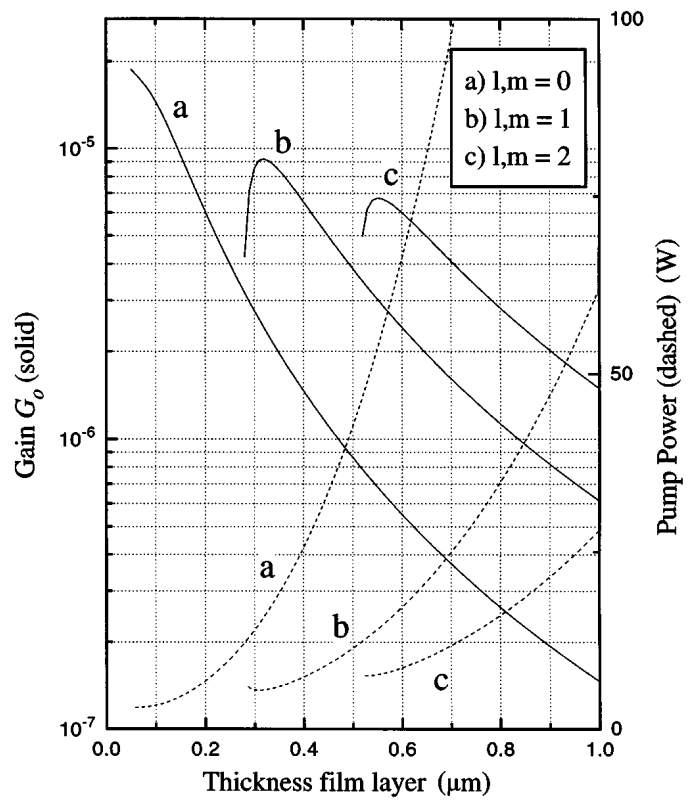

Fig. 3. Gain coefficient $G_{0}$ (solid curves) from a monolayer of benzene as a function of the film thickness with a fixed pump intensity of $10 \mathrm{MW} / \mathrm{cm}^{2}$ at the surface. Three different mode combinations are shown: $l, m=0$ (curve a), $l, m=1$ (curve b), and $l, m=2$ (curve c). The dashed curves give the pump power needed to produce these intensities. The interaction length $L=1 \mathrm{~cm}$.

The intensity inside the waveguide for the given input power is $\sim 30 \mathrm{MW} / \mathrm{cm}^{2}$ for the given thickness.

In Fig. 3 the results are shown for a monolayer of benzene [thickness $0.5 \mu \mathrm{m}, \chi^{(3)} \sim 10^{-12}$ (Ref. 13)] that is deposited onto a waveguide with $n_{\text {cladding }}=1, n_{\text {film }}=2$, and $n_{\text {substrate }}=1.46$. The intensity at the surface is kept constant at $10 \mathrm{MW} / \mathrm{cm}^{2}$. Gain $G_{0}$ is calculated for different mode combinations as indicated on the plot. Only mode combinations in which $l=m$ are chosen. Other mode combinations result in gain curves with comparable shape but with slightly smaller gain. Low-order modes $(l=m=0)$ combined with small layer thickness $(<200 \mathrm{~nm})$ result in the highest evanescent field, and therefore the total gain peaks in this region at values of the order of $10^{-5}$. The pump power necessary to produce an intensity of $10 \mathrm{MW} / \mathrm{cm}^{2}$ at the surface with a layer thickness of $150 \mathrm{~nm}$ can also be found in the plot and is $\sim 5 \mathrm{~W}$. It has been calculated that for surface stimulated Raman gain spectroscopy in a reflection geometry a gain of $10^{-8}$ can be obtained ${ }^{14}$ with a pump intensity of $100 \mathrm{MW} / \mathrm{cm}^{2}$ for a monolayer of benzene. This illustrates the advantage of using waveguides for the study of monolayers with stimulated Raman gain spectroscopy. The large increase in gain originates from the long interaction length of $1 \mathrm{~cm}$ inside the waveguide compared with the 0.5 -nm-length of the reflection geometry, leading to a gain increase of the order of $10^{7}$. The actual calculated increase in gain of $10^{4}$ is considerably smaller because in the waveguide geometry only a small part of the electric fields of the guiding modes interacts with the monolayer, whereas in the reflection geometry the monolayer interacts with the whole beam. It has been shown that even a small gain of $10^{-8}$ can be observed with a signal-to-noise ratio of $>1$ for a 1-s integration time if the double AM-FM electro-optic modulation technique is used, which achieves a great reduction in the thermal background. ${ }^{14}$ The main problem expected with the WSRGS in the detection of monolayers is the background signal from the waveguide itself. When the waveguide has any Raman resonances they will obscure the signal from the monolayer. This problem can at least partially be circumvented by the choice of proper waveguide materials or the selection of Raman bands of the thin interfacial layer with polarization properties different from those of the waveguide. Suppression techniques with different mode combinations that can be applied in WCARS are not applicable. The reason is that the overlap integral can never vanish because the mode distribution functions of both the pump and Stokes beams are squared, giving only positive contributions to the overlap integral.

In conclusion, we believe that waveguide stimulated Raman spectroscopy is a promising method for the study of thin monolayers of organic molecules. It may well be that WSRGS will outperform WCARS because of its insensitivity to the nonresonant background contribution.

We acknowledge the support provided to J. S. Kanger by the S. T. W. Technology Foundation through grant TTN11.2511.

\section{References}

1. J. F. Rabolt and J. D. Swalen, in Spectroscopy of Surfaces, R. J. H. Clark and R. E. Hester, eds., Vol. 16 of Advances in Spectroscopy (Wiley, Chichester, UK, 1988), pp. 1-36.

2. G. I. Stegeman, R. Fortenberry, C. Karaguleff, R. Moshrefzadeh, W. M. Hetherington III, N. E. Van Wyck, and J. E. Sipe, Opt. Lett. 8, 295 (1983).

3. W. M. Hetherington III, N. E. van Wyck, E. W. Koenig, G. I. Stegeman, and R. M. Fortenberry, Opt. Lett. 9, 88 (1984).

4. W. M. Hetherington III, Z. Z. Ho, E. W. Koenig, G. I. Stegeman, and R. M. Fortenberry, Proc. Soc. PhotoOpt. Instrum. Eng. 620, 102 (1986).

5. W. M. K. P. Wijekoon, E. W. Koenig, and W. M. Hetherington III, J. Phys. Chem. 97, 1065 (1993).

6. W. M. K. P. Wijekoon and W. M. Hetherington III, J. Am. Chem. Soc. 115, 2882 (1993).

7. W. P. de Boeij, J. S. Kanger, G. W. Lucassen, C. Otto, and J. Greve, Appl. Spectrosc. 47, 723 (1993).

8. T. G. Spiro, Raman Spectra and the Conformations of Biological Macromolecules, Vol. 1 of Biological Applications of Raman Spectroscopy (Wiley, New York, 1987).

9. A. K. Ghatak, K. Thyagarajan, and M. R. Shenoy, J. Lightwave Technol. LT-5, 660 (1987).

10. J. E. Sipe and G. I. Stegeman, J. Opt. Soc. Am. 69, 1676 (1979).

11. G. I. Stegeman and C. T. Seaton, J. Appl. Phys. 58, R57 (1985).

12. G. L. Eesley, Coherent Raman Spectroscopy, 1st ed. (Pergamon, Oxford, 1981), Chap. 3, p. 51.

13. P. D. Maker and R. Terhune, Phys. Rev. A 137, 801 (1965).

14. B. F. Levine and C. G. Bethea, IEEE J. Quantum Electron. QE-16, 85 (1980). 